\title{
-NOTES-
}

\section{ON THE ELASTIC DISTORTION OF A CYLINDRICAL HOLE BY A LOCALISED HYDROSTATIC PRESSURE*}

\author{
By C. J. TRANTER (Military College of Science, Shrivenham, England)
}

When a hydrostatic pressure is applied over only a small part of the length of a cylindrical hole extending through an infinite elastic solid, the stresses and displacements differ considerably from those caused by the application of this pressure over the entire length of the hole. This problem has been discussed by H. M. Westergaard ${ }^{1}$ using an approximate method but it is not easy to assess the accuracy of his numerical results. It is the purpose of the present note to give an exact solution and to compare numerical results with those given by Westergaard.

The analysis used here is a simple adaptation of that given by A. W. Rankin ${ }^{2}$ for the similar problem of a band of uniform pressure applied to a long cylindrical shaft. The numerical calculations are not so formidable as would appear at first sight and a method given by L. N. G. Filon ${ }^{3}$ for evaluating trigonometric integrals has proved very valuable in this connection. The results for the maximum radial displacement show that the approximation used by Westergaard is rather crude.

1. The analytical solution. We use cylindrical coordinates and consider the pressure loading as being given by $\sigma_{r}=-p,|z|<c, \sigma_{r}=0,|z|>c$ on the surface of the cylindrical hole $r=a$. With the usual notation ${ }^{4}$ we therefore require to find a stress function $\phi$ satisfying

$$
\nabla^{4} \phi=0, \quad r>a, \quad-\infty<z<\infty,
$$

where $\nabla^{2}$ denotes $\partial^{2} / \partial r^{2}+(1 / r)(\partial / \partial r)+\partial^{2} / \partial z^{2}$ and the boundary conditions

$$
\left.\begin{array}{c}
\sigma_{r}=\frac{\partial}{\partial z}\left\{\nu \nabla^{2}-\frac{\dot{\partial}^{2}}{\partial r^{2}}\right\} \phi=-p, \quad|z|<c, \\
=0, \quad|z|>c,
\end{array}\right\} \begin{gathered}
r=a, \\
\tau_{r z}=\frac{\partial}{\partial r}\left\{(1-\nu) \nabla^{2}-\frac{\partial^{2}}{\partial z^{2}}\right\} \phi=0, \quad-\infty<z<\infty, \quad r=a,
\end{gathered}
$$

$\nu$ being Poisson's ratio for the material of the elastic solid.

Once $\phi$ has been found, the stresses $\sigma_{r}, \tau_{r z}$ are given by the expressions shown in (2) and (3) and the remaining stresses and the radial displacement are given by

$$
\sigma_{\theta}=\frac{\partial}{\partial z}\left\{\nu \nabla^{2}-\frac{1}{r} \frac{\partial}{\partial r}\right\} \phi, \quad \sigma_{z}=\frac{\partial}{\partial z}\left\{(2-\nu) \nabla^{2}-\frac{\partial^{2}}{\partial z^{2}}\right\} \phi, \quad u=-\frac{1+\nu}{E} \cdot \frac{\partial^{2} \phi}{\partial r \partial z},
$$

$E$ being the modulus of elasticity.

* Received May 8, 1946.

${ }^{1}$ H. M. Westergaard, Kármán Anniversary Volume, 1941, p. 154.

2 A. W. Rankin, Shrink-fit stresses and deformations, Journ. Appl. Mech. 11, A77 (1944).

${ }^{3}$ L. N. G. Filon, On a quadrature formula for trigonometric integrals, Proc. Roy. Soc. Edin. 49, 38 (1928-29).

+S. Timoshenko, Theory of elasticity, McGraw-Hill Book Co., New York, 1934, p. 309. 
Following Rankin, we take

$$
\phi=\int_{0}^{\infty} R b \sin c \lambda \sin z \lambda d \lambda
$$

where $R$ is a function of $r$ only and $b$ is a function of $\lambda$. To satisfy (1) we must have

$$
\left(\frac{d^{2}}{d r^{2}}+\frac{1}{r} \frac{d}{d r}-\lambda^{2}\right)^{2} R=0,
$$

and the solution, finite as $r \rightarrow \infty$, of this equation is

$$
R=A K_{0}(\rho)+B \rho K_{1}(\rho),
$$

where $K_{0}(\rho), K_{1}(\rho)$ are Bessel functions of imaginary argument, $A$ and $B$ are constants to be found and

$$
\rho=\lambda r .
$$

Using the well known relations

$$
\left.\begin{array}{rl}
K_{0}^{\prime}(\rho) & =-K_{1}(\rho), \\
\rho K_{1}^{\prime}(\rho)+K_{1}(\rho) & =-\rho K_{0}(\rho)
\end{array}\right\}
$$

we find

$$
\nabla^{2} \phi=-2 \int_{0}^{\infty} B K_{0}(\rho) b \lambda^{2} \sin c \lambda \sin z \lambda d \lambda
$$

With

$$
\alpha=\lambda a,
$$

substitution from (9) into (3) yields

$$
\left(\tau_{r z}\right)_{r=a}=\int_{0}^{\infty}\left[-B \alpha K_{0}(\alpha)+\{2 B(1-\nu)-A\} K_{1}(\alpha)\right] b \lambda^{3} \sin c \lambda \sin z \lambda d \lambda,
$$

so that, to satisfy (3)

$$
A / B=2(1-\nu)-\alpha K_{0}(\alpha) / K_{1}(\alpha) .
$$

We also find

$$
\left(\sigma_{r}\right)_{r=a}=-\int_{0}^{\infty}\left[\{A+(2 \nu-1) B\} K_{0}(\alpha)+\left(\frac{A}{\alpha}+B \alpha\right) K_{1}(\alpha)\right] b \lambda^{3} \sin c \lambda \cos z \lambda d \lambda,
$$

and since the boundary condition (2) can be represented by

$$
\left(\sigma_{r}\right)_{r=a}=-\frac{2 p}{\pi} \int_{0}^{\infty} \frac{\sin c \lambda \cos 2 \lambda}{\lambda} d \lambda,
$$

we have

$$
b=\frac{2 p}{\pi \lambda^{4}}\left[\{A+(2 \nu-1) B\} K_{0}(\alpha)+\left(\frac{A}{\alpha}+B \alpha\right) K_{1}(\alpha)\right]^{-1} .
$$


Equations (11) and (12) yield

$$
\left.\begin{array}{l}
\pi \lambda^{4} D(\alpha) b A=2 p\left[2(1-\nu) \alpha K_{1}(\alpha)-\alpha^{2} K_{0}(\alpha)\right], \\
\pi \lambda^{4} D(\alpha) b B=2 p \alpha K_{1}(\alpha),
\end{array}\right\}
$$

where

$$
D(\alpha)=\left\{\alpha^{2}+2(1-\nu)\right\} K_{1}^{2}(\alpha)-\alpha^{2} K_{0}^{2}(\alpha) .
$$

$b A, b B$ having now been found, the expressions for the stresses and radial displacement are found to be given by

$$
\begin{aligned}
& \sigma_{r}=\frac{2 p a}{\pi r} \int_{0}^{\infty}\left[\alpha \rho K_{0}(\alpha) K_{0}(\rho)+\alpha K_{0}(\alpha) K_{1}(\rho)-\rho K_{0}(\rho) K_{1}(\alpha)\right. \\
& \left.-\left\{\rho^{2}+2(1-\nu)\right\} K_{1}(\alpha) K_{1}(\rho)\right] \frac{\sin \frac{c}{a} \alpha \cos \frac{z}{a} \alpha}{\alpha D(\alpha)} d \alpha \\
& \tau_{r z}=\frac{2 p}{\pi} \int_{0}^{\infty}\left[\alpha K_{0}(\alpha) K_{1}(\rho)-\rho K_{0}(\rho) K_{1}(\alpha)\right] \frac{\sin \frac{c}{a} \alpha \sin \frac{z}{a} \alpha}{D(\alpha)} d \alpha \\
& \sigma_{\theta}=-\frac{2 p a}{\pi r} \int_{0}^{\infty}\left[\alpha K_{0}(\alpha) K_{1}(\rho)+(2 \nu-1) \rho K_{0}(\rho) K_{1}(\alpha)\right. \\
& \left.-2(1-\nu) K_{1}(\alpha) K_{1}(\rho)\right] \frac{\sin \frac{c}{a} \alpha \cos \frac{\tilde{z}}{a} \alpha}{\alpha D(\alpha)} d \alpha \\
& \sigma_{z}=-\frac{2 p}{\pi} \int_{0}^{\infty}\left[\alpha K_{0}(\alpha) K_{0}(\rho)+2 K_{0}(\rho) K_{1}(\alpha)\right. \\
& \left.-\rho K_{1}(\alpha) K_{1}(\rho)\right] \frac{\sin \frac{c}{a} \alpha \cos \frac{z}{a} \alpha}{D(\alpha)} d \alpha \\
& \frac{E u}{1+\nu}=-\frac{2 p a}{\pi} \int_{0}^{\infty}\left[\alpha K_{0}(\alpha) K_{1}(\rho)-\rho K_{0}(\rho) K_{1}(\alpha)\right. \\
& \left.-2(1-\nu) K_{1}(\alpha) K_{1}(\rho)\right] \frac{\sin \frac{c}{a} \alpha \cos \frac{z}{a} \alpha}{\alpha D(\alpha)} d \alpha
\end{aligned}
$$

2. Numerical results for the maximum radial displacement at $r=a$. When $r=a$, $\rho=\alpha$ and the greatest displacement occurs when $z=0$, so that we have

$$
\frac{E\left(u_{\max }\right)_{r=a}}{1+\nu}=\frac{4 p a(1-\nu)}{\pi} \int_{0}^{\infty} \frac{K_{1}^{2}(\alpha)}{\alpha D(\alpha)} \sin \frac{c}{a} \alpha d \alpha .
$$


If the pressure $p$ acts over the entire length of the hole, the displacement $\left(u^{\prime}\right)_{r=a}$ is given by

$$
\frac{E\left(u^{\prime}\right)_{r=a}}{1+\nu}=p a,
$$

so that

$$
\frac{\left(u_{\max }\right)_{r=a}}{\left(u^{\prime}\right)_{r=a}}=\frac{4(1-\nu)}{\pi} \int_{0}^{\infty} \frac{K_{1}^{2}(\alpha)}{\alpha D(\alpha)} \sin \frac{c}{a} \alpha d \alpha .
$$

The numerical work was performed with $\nu=0.3$ and, above $\alpha=12$, it was found that the first three terms of the asymptotic expansion of $K_{1}^{2}(\alpha) / \alpha D(\alpha)$, viz.,

$$
\frac{K_{1}^{2}(\alpha)}{\alpha D(\alpha)} \sim \frac{1}{\alpha^{2}}-\frac{0.4}{\alpha^{2}}-\frac{0.965}{\alpha^{4}}
$$

gave an adequate representation. Integration by parts then leads to

$$
\begin{aligned}
\int_{12}^{\infty} \frac{K_{1}^{2}(\alpha)}{\alpha D(\alpha)} \sin \frac{c}{a} \alpha d \alpha= & {\left[.08176+.01340 \frac{c^{2}}{a^{2}}\right] \sin \frac{12 c}{a}-.01778 \frac{c}{a} \cos \frac{12 c}{a} } \\
& +.2 \frac{c^{2}}{a^{2}}\left[\frac{\pi}{2}-\operatorname{Si}\left(\frac{12 c}{a}\right)\right]-\left[1+.16083 \frac{c^{2}}{a^{2}}\right] \frac{c}{a} \operatorname{Ci}\left(\frac{12 c}{a}\right),
\end{aligned}
$$

where

$$
\operatorname{Si}(x)=\int_{0}^{x} \frac{\sin x}{x} d x, \quad \operatorname{Ci}(x)=-\int_{x}^{\infty} \frac{\cos x}{x} d x .
$$

The evaluation of the integral in equation (16) from $\alpha=0$ to $\alpha=12$ was performed as follows. The funotion $K_{1}^{2}(\alpha) / \alpha D(\alpha)$ was computed at intervals of $\alpha=0.2$ from $\alpha=0$ to $\alpha=2$ and at intervals of $\alpha=0.5$ from $\alpha=2$ to $\alpha=12$. The integral was then evaluated by a method due to Filon ${ }^{5}$ in which Simpson's rule is replaced by the formula

$$
\int_{A}^{B} F(x) \sin k x d x=h\left[\alpha\{F(A) \cos k A-F(B) \cos k B\}+\beta S_{2 s}+\gamma S_{2 s-1}\right],
$$

where the range of integration is divided into intervals of length $h, S_{2}$ is the sum of all the even ordinates of the curve $y=F(x) \sin k x$ between $A$ and $B$ inclusive less half the first and last ordinates, $S_{2 s-1}$ is the sum of all the odd ordinates, and $\alpha, \beta, \gamma$ are given in terms of $\psi=h k$ by

$$
\begin{gathered}
\alpha=\frac{1}{\psi}+\frac{\sin \psi \cos \psi}{\psi^{2}}-\frac{2 \sin ^{2} \psi}{\psi^{3}}, \quad \beta=2\left[\frac{1+\cos ^{2} \psi}{\psi^{2}}-\frac{2 \sin \psi \cos \psi}{\psi^{3}}\right], \\
\gamma=4\left[\frac{\sin \psi}{\psi^{3}}-\frac{\cos \psi}{\psi^{2}}\right] .
\end{gathered}
$$

- Filon, loc. cit. 
This formula holds even when $k$ is large, provided that the function $F(x)$ can be fitted with reasonable accuracy over the range $2 h$ by parabolic arcs.

To a void an infinity at the origin, the integral actually evaluated was

$$
I=\int_{0}^{12}\left[\frac{1}{1.4 \alpha}-\frac{K_{1}^{2}(\alpha)}{\alpha D(\alpha)}\right] \sin \frac{c}{a} \alpha d \alpha,
$$

and when this had been found, the required integral was given by

$$
\frac{1}{1.4} \mathrm{Si}\left(\frac{12 c}{a}\right)-I \text {. }
$$

As a check that the substitution of the asymptotic series did not lead to unacceptable errors, the range of integration was also divided into 0 to 10,10 to infinity and the infinite integral was similarly computed on this basis. Little extra work was involved and excellent agreement was obtained.

The results are shown below, together with those given by the approximate analysis by Westergaard. It is seen that even his second approximation is quite crude.

\begin{tabular}{c|c|c|c}
\hline \hline \multirow{3}{*}{$\frac{c}{a}$} & \multicolumn{2}{|c|}{ Values of $\left(u_{\max }\right)_{r-a} /\left(u^{\prime}\right)_{r-a}$} \\
\cline { 2 - 3 } & Westergaard & \multirow{2}{*}{ Present Method } \\
\cline { 2 - 4 } & First Approximation & Second Approximation & \\
\hline 0.25 & 0.557 & 0.537 & 0.450 \\
0.50 & 0.806 & 0.770 & 0.633 \\
\hline
\end{tabular}

\section{ON THE REPEATED INTEGRALS OF BESSEL FUNCTIONS*}

By J. C. JAEGER (University of Tasmania)

It is well known that

$$
L\left\{\frac{n J_{n}(t)}{t}\right\}=\left[\left(p^{2}+1\right)^{1 / 2}-p\right]^{n}, \quad n>0,
$$

and

$$
L\left\{J_{n}(t)\right\}=\frac{\left[\left(p^{2}+1\right)^{1 / 2}-p\right]^{n}}{\left(p^{2}+1\right)^{1 / 2}}, \quad n \geqq 0
$$

* Received Jan. 25, 1946. 